\title{
Article \\ Uncertain Hypergraphs: A Conceptual Framework and Some Topological Characteristics Indexes
}

\author{
Jin Peng ${ }^{1}\left(\mathbb{D}\right.$, Bo Zhang ${ }^{2, *(\mathbb{C})}$ and Kiki Ariyanti Sugeng ${ }^{3 \oplus \mathbb{C}}$
}

1 School of Mathematics and Statistics, Institute of Uncertain Systems, Huanggang Normal University, Huanggang 438000, China; peng@hgnu.edu.cn

2 School of Statistics and Mathematics, Zhongnan University of Economics and Law, Wuhan 430073, China

3 Faculty of Mathematics and Science, University of Indonesia, Depok 16424, Indonesia; kiki@sci.ui.ac.id

* Correspondence: bozhang@zuel.edu.cn

check for updates

Citation: Peng, J.; Zhang, B.; Sugeng, K.A. Uncertain Hypergraphs: A Conceptual Framework and Some Topological Characteristics Indexes. Symmetry 2022, 14, 330. https:// doi.org/10.3390/sym14020330

Academic Editors: Tingqing Ye, Waichon Lio and Baoding Liu

Received: 4 January 2022

Accepted: 2 February 2022

Published: 5 February 2022

Publisher's Note: MDPI stays neutral with regard to jurisdictional claims in published maps and institutional affiliations.

Copyright: (c) 2022 by the authors. Licensee MDPI, Basel, Switzerland. This article is an open access article distributed under the terms and conditions of the Creative Commons Attribution (CC BY) license (https:// creativecommons.org/licenses/by/ $4.0 /)$.

\begin{abstract}
In practical applications of hypergraph theory, we are usually surrounded by the state of indeterminacy. This paper employs uncertainty theory to address indeterministic information. We initially put forward the idea of an uncertain hypergraph by combining hypergraph theory with uncertainty theory in order to provide a useful tool to deal with a variety of uncertain complex systems and to create a new interdisciplinary research field. The main focus of this paper is to propose a conceptual framework of uncertain hypergraphs and to study the operations of uncertain hypergraphs. Moreover, some topological indexes are proposed to describe the characteristics of the structures of uncertain hypergraph. Additionally, some further research directions are discussed.
\end{abstract}

Keywords: graph; uncertain graph; uncertain hypergraph; uncertain system; uncertainty theory

\section{Introduction}

Graph theory has a history of more than 280 years, beginning when mathematician Euler first used a graph approach to solve the problem of the Seven Bridges of Königsberg in 1736. It commenced its formal development during the second half of the 19th century, and substantial growth has been witnessed during the last 100 years. Until now, graphs have been extensively used in domains including mathematics and computer science as well as various other fields, ranging from neuroscience to social network analysis.

However, graphs have a major limitation in that they cannot represent complex multi-ary relations among things but only support binary relations between pairs of things in a system. In real life, the multi-ary relationships extending unary and binary relationships exist commonly in a complex system. N-ary relations describe relations of any arity, including unary, binary, ternary, etc. As a generalization of traditional graph theory (see, for example, Bondy and Murty [1]), the hypergraph was introduced by Berge [2] to describe the complex systems of multi-ary relationships other than binary relationships. The co-occurrence relation can be represented by a hyperedge that connects two or more vertices in a hypergraph. Since then, many popular topics of hypergraphs have been studied, such as connectivity (Dankelmann and Meierling [3], Zhao and Meng [4]), degree (Frankl [5], Hàn et al. [6]), matching problems (Yu et al. [7], Lu et al. [8]) and so forth.

In practice, indeterminacy is inevitable due to the lack of observed data. Sometimes, whether an edge exists cannot be completely determined. Then, how does one address such indeterministic information? Some researchers thought that whether an edge existed could be described as a random variable. As a result, probability theory (Kolmogorov [9]) was introduced into graph theory, and the random graph was defined by Erdös and Rényi [10,11] and Gilbert [12] at nearly the same time. Subsequently, the random hypergraph has been studied by many scholars. For instance, Cooper [13] studied the problem of asymptotical descriptions of the adjacency eigenvalues of random and complete uniform hypergraphs. Semenov and Shabanov [14] addressed the weak chromatic 
numbers of random hypergraphs. Liu and Zhao [15] investigated the upper tail problem for hypergraphs.

It is universally acknowledged that probability theory can be used to describe indeterminate quantities only after we can obtain sufficient data to obtain a probability distribution close enough to the real frequency. However, in real life, we usually have no access to sufficient data. In this case, we have no choice but to invite some domain experts in order to obtain belief degrees for quantities with indeterministic information. Then, how can we handle belief degrees? As a development of fuzzy set theory (Zadeh [16]), some fuzziologists believed such indeterminacy could be interpreted as fuzziness and attempted to introduce fuzzy set theory to graph theory. Subsequently, the fuzzy graph (Rosenfeld [17]) was proposed, and a variety of work on fuzzy graphs and fuzzy hypergraphs has been carried out. For example, Wang and Gong [18]) introduced a method to formulate granular structure models by using a fuzzy hypergraph. Luqman et al. [19] developed novel generalized fuzzy hypergraphs and presented an application through a complex $q$ rung orthopair fuzzy hypergraph. Sarwar et al. [20] proposed a new framework of bipolar fuzzy soft hypergraphs and introduced various methods for construction of bipolar fuzzy soft hypergraphs. There are many papers written on the subject of fuzzy graphs and fuzzy hypergraphs; the interested readers may refer to Akram and Luqman [21].

Unfortunately, it is not suitable to regard every indeterministic phenomenon as a random phenomenon or fuzzy phenomenon, especially when the indeterministic phenomenon comes from experts' belief degrees. In order to model such belief degrees rationally, axiomatic uncertainty theory was founded by Liu [22] in 2007 and then refined by Liu [23] in 2010. Up to now, uncertainty theory has developed rapidly and has been widely applied in management science, computing science, reliability systems and other related fields. In terms of graph theory, Gao and Gao [24] defined the concept of the uncertain graph. Almost at the same time, Zhang and Peng studied the Euler tour problem (Zhang and Peng [25]), matching problem (Zhang and Peng [26]) and connectivity (Zhang and Peng [27]) of uncertain graphs. In addition, regularity (Gao [28]) and edge-connectivity (Gao and Qin [29]) of uncertain graphs have also been studied. In most natural and social systems, there exist both complexity and uncertainty. Therefore, it is necessary to develop understanding of the properties of hypergraphs with experts' belief degrees.

Inspired by the above discussion, this article mainly focuses on the following two issues: First, how can we model a hypergraph with experts' belief degrees? Second, what is the topology structure of a hypergraph with experts' belief degrees? To answer the questions, the concept of uncertain hypergraph is proposed. Then, representations of an uncertain hypergraph are discussed. Apart from that, operations of uncertain hypergraphs are studied based on complement, union and joint. Additionally, some topological indexes are proposed to further describe the characteristics of the structures of uncertain hypergraphs.

The rest of the paper is organized as follows: Firstly, we review the relevant basic knowledge, including hypergraph theory, uncertainty theory and uncertain graph theory in Section 2. Next, in Section 3, some new concepts of uncertain hypergraphs are introduced and are illustrated by some examples to make sense. Then, operations of uncertain hypergraphs are investigated in Section 4. Moreover, some topological indexes are given to describe the uncertain structure of multi-ary relationships for uncertain hypergraphs in Section 5. Finally, the paper ends with conclusions and a summary of future work.

\section{Preliminaries}

\subsection{Hypergraphs}

The concept of a hypergraph is mathematically a generalization of a graph. In a hypergraph, one edge can join any number of vertices, while each edge can only join two vertices, at most, in a classical graph. Hence, hypergraph models describe more general and complex types of relations than graph models do. 
In this section, we introduce basic notions about hypergraphs. We suppose that readers are familiar with the basic knowledge of graph theory. As hypergraphs are an important generalization of ordinary graphs, many of the definitions of graphs can be extended to hypergraphs in a nearly verbatim way.

Taking Bergé [2] and Bretto [30] as references, we give the following definition:

Definition 1. A hypergraph $H$ is a pair $H=(V, E)$, where $V$ is a finite set of elements and $E$ is a family of subsets of $V$. Customarily, $V=\left\{v_{1}, v_{2}, \cdots, v_{n}\right\}$ is called the vertex set of $H$, denoted by $V(H)$ sometimes for clarity, and $E=\left\{e_{i} \mid e_{i}=\left\{v_{i_{1}}, v_{i_{2}}, \cdots, v_{i_{n_{i}}}\right\}\right\}$ is called the hyperedge set of $H$, denoted by $E(H)$ sometimes for clearness.

We say that the cardinality $|V|$ of $V$ is the order of the hypergraph $H=(V, E)$, and the cardinality $|E|$ of $E$ is the size of the hypergraph $H=(V, E)$. It can be seen from the above definition that a hyperedge in a hypergraph may connect any number of vertices. The hyperedge set $E$ is a subset of the power set $\mathcal{P}(V)$ of the vertex set $V$ in a hypergraph. A vertex $x$ in a hypergraph is isolated if $x \in V-\bigcup_{i \in|E|} V\left(e_{i}\right)$. A hyperedge $e \in E$ such that $|V(e)|=1$ is a loop. Now we consider the question: How can we visually represent the hypergraph in a reasonable way? There are two different kinds of representations; one is a geometrical method and the other is an algebraical method.

Example 1. Let $H=(V, E)$ be a hypergraph with $V=\left\{v_{1}, v_{2}, v_{3}, v_{4}, v_{5}, v_{6}, v_{7}, v_{8}\right\}$ and $E=\left\{e_{1}, e_{2}, e_{3}, e_{4}, e_{5}, e_{6}\right\}=\left\{\left\{v_{3}, v_{4}, v_{5}\right\},\left\{v_{5}, v_{8}\right\},\left\{v_{6}, v_{7}, v_{8}\right\},\left\{v_{7}\right\},\left\{v_{1}, v_{2}\right\},\left\{v_{2}, v_{3}, v_{7}\right\}\right\}$. Then $H$ has 8 vertices and 6 hyperedges. The hypergraph $H$ is graphically illustrated in Figure 1.

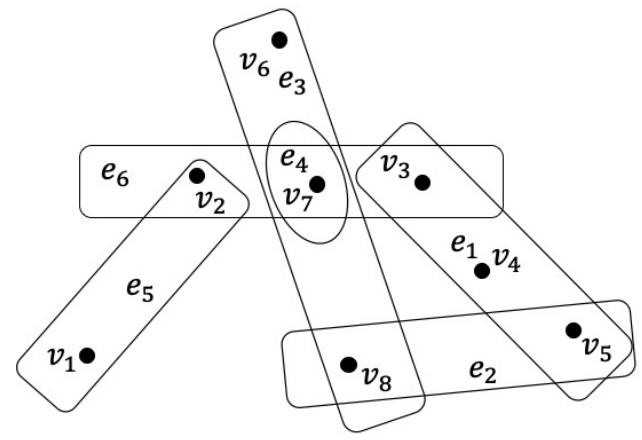

Figure 1. A hypergraph.

Example 2. Suppose eight members comprise a research team $V=\left\{v_{1}, v_{2}, \cdots, v_{8}\right\}$. Now consider the collaborative relationship between them for published papers. The set $E=\left\{e_{1}, e_{2}, e_{3}, e_{4}, e_{5}\right\}$ means a total of 5 articles have been published, and the authors of each article are $e_{1}=\left\{v_{1}, v_{2}, v_{3}, v_{4}\right\}$, $e_{2}=\left\{v_{2}, v_{3}, v_{4}\right\}, e_{3}=\left\{v_{1}, v_{5}, v_{7}\right\}, e_{4}=\left\{v_{6}, v_{7}\right\}$ and $e_{5}=\left\{v_{8}\right\}$, respectively. Therefore, the research team's paper collaboration hypergraph in a knowledge network analysis is $H=(V, E)$ with 8 vertices and 5 hyperedges, which is shown in Figure 2. In fact, the hypergraph $H$ may stand for the kinds of coauthor relationships among 8 authors.

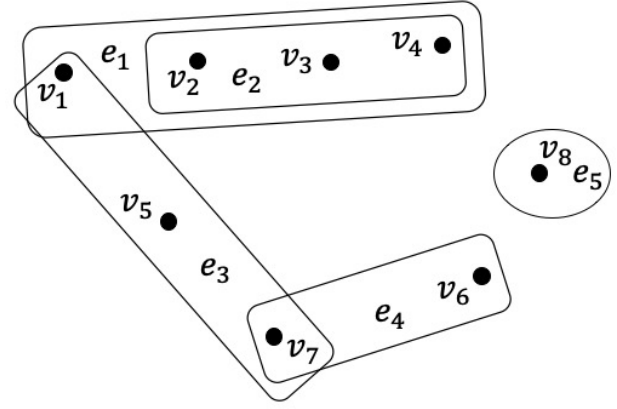

Figure 2. A hypergraph of research team. 
Example 3. Nowadays, WeChat (or QQ) is one of the most popular social media platforms for Chinese social network users. This new form of media, instead of telephone and email, is powerfully changing the way of social communication. Consider somebody's contact objects and private groups of WeChat. Assume that $V=\left\{v_{1}, v_{2}, \cdots, v_{12}\right\}$ and $E=\left\{e_{1}, e_{2}, e_{3}, e_{4}, e_{5}\right\}=$ $\left\{\left\{v_{1}, v_{2}, v_{3}\right\},\left\{v_{4}, v_{5}, v_{9}, v_{10}\right\},\left\{v_{2}, v_{3}, v_{5}, v_{6}, v_{7}\right\},\left\{v_{3}, v_{7}, v_{8}, v_{11}\right\},\left\{v_{10}, v_{11}, v_{12}\right\}\right\}$. Then H has 12 vertices, which represent contact objects in his or her WeChat address list, and 5 hyperedges, which represent his or her private group in WeChat. The hypergraph $H=(V, E)$ has the visualization depicted in Figure 3.

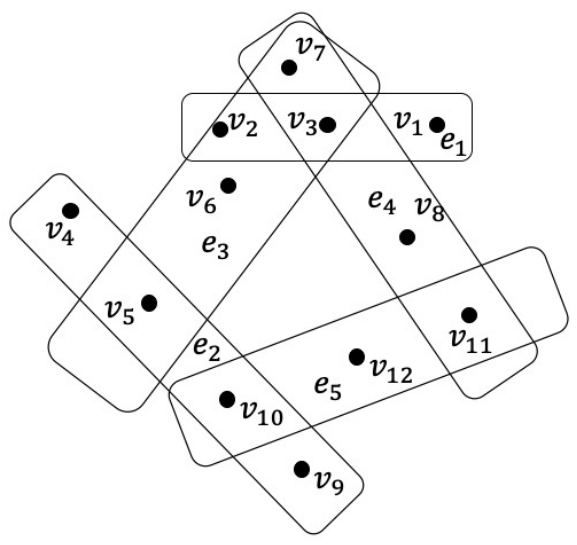

Figure 3. A hypergraph of social network.

\subsection{Uncertainty Theory and Uncertain Graph Theory}

Uncertainty theory was invented by Liu [22] to rationally model belief degrees.

Definition 2. (Liu [22]) Let $\mathcal{L}$ be a $\sigma$-algebra on a nonempty set $\Gamma$. An arbitrary set function $\mathcal{M}: \mathcal{L} \rightarrow[0,1]$ is called an uncertain measure if it satisfies the following three axioms:

Axiom 1. $\mathcal{M}\{\Gamma\}=1$ for the universal set $\Gamma$.

Axiom 2. $\mathcal{M}\{\Lambda\}+\mathcal{M}\left\{\Lambda^{c}\right\}=1$ for any event $\Lambda$.

Axiom 3. For every countable sequence of events $\Lambda_{1}, \Lambda_{2}, \cdots$, we have

$$
\mathcal{M}\left\{\bigcup_{i=1}^{\infty} \Lambda_{i}\right\} \leq \sum_{i=1}^{\infty} \mathcal{M}\left\{\Lambda_{i}\right\} .
$$

The triplet $(\Gamma, \mathcal{L}, \mathcal{M})$ is said to be an uncertainty space. An uncertain variable (Liu [22]) is defined as a measurable function $\xi$ from an uncertainty space to the set of real numbers. The product uncertain measure was defined by Liu [31] in 2009, thus producing the fourth axiom of uncertainty theory.

Axiom 4. Let $\left(\Gamma_{k}, \mathcal{L}_{k}, \mathcal{M}_{k}\right)$ be uncertainty spaces for $k=1,2, \cdots$. The product uncertain measure $\mathcal{M}$ is an uncertain measure satisfying

$$
\mathcal{M}\left\{\prod_{k=1}^{\infty} \Lambda_{k}\right\}=\bigwedge_{k=1}^{\infty} \mathcal{M}_{k}\left\{\Lambda_{k}\right\},
$$

where $\Lambda_{k}$ are arbitrarily chosen events from $\mathcal{L}_{k}$ for $k=1,2, \cdots$, respectively.

Definition 3. (Liu [22]) The uncertainty distribution of an uncertain variable $\xi$ is defined by

$$
\Phi(x)=\mathcal{M}\{\xi \leq x\}
$$

for any real number $x$.

An uncertain variable is called Boolean if it takes the values of either 0 or 1 . That is to say, a Boolean uncertain variable can be expressed as follows: 


$$
\xi= \begin{cases}1 & \text { with uncertain measure } a \\ 0 & \text { with uncertain measure } 1-a\end{cases}
$$

where $a \in[0,1]$ is a real number.

Definition 4. (Liu [31]) Let $\xi$ be an uncertain variable with uncertainty distribution $\Phi$. Then its entropy is defined by

$$
H(\xi)=\int_{-\infty}^{+\infty} S(\Phi(x)) d x
$$

where $S(t)=-t \ln t-(1-t) \ln (1-t)$.

As discussed in the introduction, many scholars have paid attention to research topics on uncertain graphs. We begin by recalling the most standard definitions of uncertain graphs (Zhang and Peng [25]). Note that an uncertain graph can be redefined by the method of set and mapping in the following way:

Definition 5. An uncertain graph is defined as a triple system $G=(V, E, \varphi)$ where $V$ is the vertex set of $G, E(\subseteq V \times V)$ is the edge set of $G$ and $\varphi: E \rightarrow[0,1]$ is a belief degree function in which $\varphi(e)$ assigns the uncertain measure of the existence of the edge $e \in E$.

It follows from Definition 5 that in an uncertain graph, the vertices are all predetermined, while edges are uncertain. The existence possibility of an edge is described by uncertain measure. Generally, a Boolean uncertain variable $\xi_{i}$ can be used to describe the existence of edge $e_{i}$. That is, $\varphi\left(e_{i}\right)=\mathcal{M}\left\{\xi_{i}=1\right\}$ indicates the existence possibility of $e_{i}$, where $\xi_{i}=1$ means edge $e_{i}$ exists. Apparently, the larger the value of $\varphi\left(e_{i}\right)$, the more the true it is that the edge $e_{i}$ exists. In particular, $\varphi\left(e_{i}\right)=1$ means that the edge $e_{i}$ exists completely; on the contrary, $\varphi\left(e_{i}\right)=0$ means that the edge $e_{i}$ does not exist. For more research on uncertainty theory and uncertain graphs, the interested readers may refer to Liu [32] and Zhang and Peng [25].

Example 4. Let $G=(V, E)$ be an uncertain graph with vertex set $V=\left\{v_{1}, v_{2}, v_{3}, v_{4}, v_{5}, v_{6}\right\}$, edge set $E=\left\{e_{1}, e_{2}, e_{3}, e_{4}, e_{5}, e_{6}, e_{7}, e_{8}, e_{9}\right\}$, belief degree function on $E$ as follows:

$$
E=\left\{\left\{v_{1}, v_{2}\right\},\left\{v_{2}, v_{3}\right\},\left\{v_{3}, v_{4}\right\},\left\{v_{4}, v_{5}\right\},\left\{v_{5}, v_{6}\right\},\left\{v_{6}, v_{1}\right\},\left\{v_{1}, v_{4}\right\},\left\{v_{2}, v_{5}\right\},\left\{v_{3}, v_{6}\right\}\right\}
$$

and

$$
\begin{gathered}
\varphi\left(e_{1}\right)=0.91, \varphi\left(e_{2}\right)=0.92, \varphi\left(e_{3}\right)=0.93, \varphi\left(e_{4}\right)=0.94, \varphi\left(e_{5}\right)=0.95, \\
\varphi\left(e_{6}\right)=0.96, \varphi\left(e_{7}\right)=0.97, \varphi\left(e_{8}\right)=0.98 \text { and } \varphi\left(e_{9}\right)=0.99
\end{gathered}
$$

Then $G=(V, E, \varphi)$ is an uncertain graph with the uncertain adjacency matrix

$$
A=\left(\begin{array}{cccccc}
0 & 0.91 & 0 & 0.97 & 0 & 0.96 \\
0.91 & 0 & 0.92 & 0 & 0.98 & 0 \\
0 & 0.92 & 0 & 0.93 & 0 & 0.99 \\
0.97 & 0 & 0.93 & 0 & 0.94 & 0 \\
0 & 0.98 & 0 & 0.94 & 0 & 0.95 \\
0.96 & 0 & 0.99 & 0 & 0.95 & 0
\end{array}\right) .
$$

\section{Uncertain Hypergraphs}

Despite the fact that a hypergraph can only represent the determining multi-ary relations (including binary relations and unary relations) in a complex system, it cannot be used to deal with the uncertain multi-ary relations in a complex system under the presence of indeterminacy. 
We have introduced the fundamental concepts of hypergraph theory and uncertainty theory as above-mentioned. We now aim to combine the two by exploring the notions of uncertain hypergraphs.

In this section, we introduce the basic notions about uncertain hypergraphs. From a theoretical point of view, on one hand, an uncertain hypergraph is the generation of a hypergraph stated in Section 1; on the other hand, an uncertain hypergraph can be considered the extension of an uncertain graph, which will be reviewed in the coming subsection.

\subsection{Concept of Uncertain Hypergraphs}

As the generalization of a hypergraph, an uncertain hypergraph is defined as follows:

Definition 6. An uncertain hypergraph is defined as a triple system $H=(V, E, \psi)$ where $V$ is the vertex set, $E$ is a family of subsets of $V$ and $\psi: E \rightarrow[0,1]$ is a set function in which $\psi(e)$ assigns the uncertain measure of the existence of the hyperedge $e \in E$. Correspondingly, $\underline{H}=(V, E)$ is called the underlying hypergraph of uncertain hypergraph $H$ and $\psi$ is known as the hyperedge assignment function of uncertain hypergraph $H$.

A simple hypergraph is a hypergraph having no loops and multiple hyperedges among (or between) a set of vertices. Every hypergraph in this paper is a simple uncertain hypergraph.

Remark 1. When the hyperedge set $E(\in \mathcal{P}(V))$ of $H$ degrades into the classical edge set $E(\subseteq$ $V \times V)$, an uncertain hypergraph degenerates to an uncertain graph. Thus, an uncertain hypergraph is an extension of an uncertain graph.

Remark 2. When the hyperedge assignment function $\psi \equiv 1$ of $H$, an uncertain hypergraph can be regarded as an ascertained hypergraph. Thus, an uncertain hypergraph is also an extension of a determined hypergraph.

Remark 3. When each hyperedge in $E$ is an ordered set with a known head and tail, an uncertain hypergraph will become an uncertain directed hypergraph, or uncertain dihypergraph. Thus, an uncertain dihypergraph is both an extension of an uncertain digraph and an extension of a determined dihypergraph.

Example 5. Let $H=(V, E)$ be a hypergraph with vertices $V=\left\{v_{1}, v_{2}, v_{3}, v_{4}, v_{5}, v_{6}\right\}$ and hyperedges $E=\left\{e_{1}, e_{2}, e_{3}, e_{4}\right\}=\left\{\left\{v_{1}, v_{2}, v_{3}\right\},\left\{v_{2}, v_{3}\right\},\left\{v_{2}, v_{4}, v_{5}\right\},\left\{v_{6}\right\}\right\}$. Define $\psi\left(e_{1}\right)=$ $0.85, \psi\left(e_{2}\right)=0.9, \psi\left(e_{3}\right)=0.95$ and $\psi\left(e_{4}\right)=1$. Then $H=(V, E, \psi)$ is an uncertain hypergraph depicted in Figure 4.

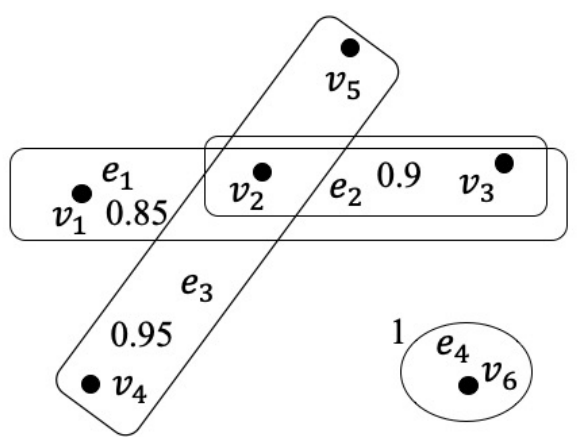

Figure 4. An uncertain hypergraph.

\subsection{Representation of Uncertain Hypergraphs}

Assume $H=(V, E, \psi)$ is an uncertain hypergraph of order $n$ with $V=\left\{v_{1}, v_{2}, \cdots, v_{n}\right\}$ $E=\left(e_{1}, e_{2}, \cdots, e_{m}\right)$ and $\psi(E)=\left(\psi\left(e_{1}\right), \psi\left(e_{2}\right), \cdots, \psi\left(e_{m}\right)\right)$. How can we visually represent 
the uncertain hypergraph in a reasonable way? There are two different kinds of representations aside from the set method as stated in the definition; one is a geometrical method and the other is an algebraical method.

In a geometrical way, we can put in the uncertain measure on each hyperedge based on the underlying hypergraph $H=(V, E)$ (see, for example, Figure 4). In an algebraical way, we can use two types of uncertain hyper-adjacency matrices. In other words, each uncertain hypergraph can be represented by the minimum hyper-adjacency matrix or the maximum hyper-adjacency matrix.

Definition 7. The minimum hyper-adjacency matrix of an uncertain hypergraph $H=(V, E, \psi)$ of order $n$ is defined as

$$
\underline{A}=\left(\begin{array}{cccc}
\alpha_{11} & \alpha_{12} & \cdots & \alpha_{1 n} \\
\alpha_{21} & \alpha_{22} & \cdots & \alpha_{2 n} \\
\vdots & \vdots & \ddots & \vdots \\
\alpha_{n 1} & \alpha_{n 2} & \cdots & \alpha_{n n}
\end{array}\right)
$$

where $\alpha_{i j}$ represent that the hyperedges containing vertices $v_{i}$ and $v_{j}$ exist with uncertain measures $\alpha_{i j}=\min \left\{\psi(e) \mid v_{i}, v_{j} \in e, e \in E\right\}, i, j=1,2, \cdots, n$, respectively.

Definition 8. The maximum hyper-adjacency matrix of an uncertain hypergraph $H=(V, E, \psi)$ of order $n$ is defined as

$$
\bar{A}=\left(\begin{array}{cccc}
\alpha_{11} & \alpha_{12} & \cdots & \alpha_{1 n} \\
\alpha_{21} & \alpha_{22} & \cdots & \alpha_{2 n} \\
\vdots & \vdots & \ddots & \vdots \\
\alpha_{n 1} & \alpha_{n 2} & \cdots & \alpha_{n n}
\end{array}\right)
$$

where $\alpha_{i j}$ represent that the hyperedges contain vertices $v_{i}$ and $v_{j}$ exist with uncertain measures $\alpha_{i j}=\max \left\{\psi(e) \mid v_{i}, v_{j} \in e, e \in E\right\}, i, j=1,2, \cdots, n$, respectively.

Example 6. Let $H=(V, E, \psi)$ be the uncertain hypergraph in Example 5. Then the minimum hyper-adjacency matrix and the maximum hyper-adjacency matrix are respectively

$$
\begin{aligned}
\underline{A} & =\left(\begin{array}{cccccc}
0 & 0.85 & 0.85 & 0 & 0 & 0 \\
0.85 & 0 & 0.85 & 0.95 & 0.95 & 0 \\
0.85 & 0.85 & 0 & 0 & 0 & 0 \\
0 & 0.95 & 0 & 0 & 0.95 & 0 \\
0 & 0.95 & 0 & 0.95 & 0 & 0 \\
0 & 0 & 0 & 0 & 0 & 1
\end{array}\right) \text { and } \\
\bar{A} & =\left(\begin{array}{cccccc}
0 & 0.85 & 0.85 & 0 & 0 & 0 \\
0.85 & 0 & 0.9 & 0.95 & 0.95 & 0 \\
0.85 & 0.9 & 0 & 0 & 0 & 0 \\
0 & 0.95 & 0 & 0 & 0.95 & 0 \\
0 & 0.95 & 0 & 0.95 & 0 & 0 \\
0 & 0 & 0 & 0 & 0 & 1
\end{array}\right) .
\end{aligned}
$$

\subsection{Variations of Uncertain Hypergraphs}

The above-mentioned uncertain hypergraph is considered an adjacency uncertain hypergraph. We can now consider the other type of uncertain hypergraph, that is, the incidence uncertain hypergraph. 
Definition 9. An incidence uncertain hypergraph is defined as a triple system $H=\left(V, E, \psi^{\prime}\right)$ where $V$ is the vertex set, $E$ is the hyperedge set of $H$ and $\psi^{\prime}: V \times E \rightarrow[0,1]$ is a set function in which $\psi^{\prime}((v, e))$ assigns the uncertain measure of the incidence existence between vertex $v \in V$ and hyperedge $e \in E$. Correspondingly, $\underline{H}=(V, E)$ is still called the underlying hypergraph of uncertain hypergraph $H$ and $\psi^{\prime}$ is known as the incidence assignment function of uncertain hypergraph $H$.

Assume $H=(V, E)$ is a hypergraph of order $n$ and size $m$ with $V=\left\{v_{1}, v_{2}, \cdots, v_{n}\right\}$ and $E=\left\{e_{1}, e_{2}, \cdots, e_{m}\right\}$. Each hypergraph can be represented by the incidence matrix as stated in Definition 9.

Definition 10. A hypergraph $H=(V, E)$ of order $n$ and size $m$ is said to be of uncertain incidence if it has uncertain incidence matrix $B=\left(\beta_{i j}\right)_{n \times m}$ as follows:

$$
B=\left(\begin{array}{cccc}
\beta_{11} & \beta_{12} & \cdots & \beta_{1 m} \\
\beta_{21} & \beta_{22} & \cdots & \beta_{2 m} \\
\vdots & \vdots & \ddots & \vdots \\
\beta_{n 1} & \beta_{n 2} & \cdots & \beta_{n m}
\end{array}\right)
$$

where $\beta_{i j} \in[0,1]$ represents that the hyperedge $e_{j}$ containing vertex $v_{i}$ exists with uncertain measure $\beta_{i j}, i=1,2, \cdots, n ; j=1,2, \cdots, m$, respectively.

As a matter of fact, if $\beta_{i j} \in\{0,1\}$, then the above-mentioned uncertain incidence matrix will be degraded to the traditional incidence matrix.

Example 7. Let $H=(V, E)$ be the hypergraph in Example 1. Then the incidence matrix is

$$
C=\left(\begin{array}{llllll}
0 & 0 & 0 & 0 & 1 & 0 \\
0 & 0 & 0 & 0 & 1 & 1 \\
1 & 0 & 0 & 0 & 0 & 1 \\
1 & 0 & 0 & 0 & 0 & 0 \\
1 & 1 & 0 & 0 & 0 & 0 \\
0 & 0 & 1 & 0 & 0 & 0 \\
0 & 0 & 1 & 1 & 0 & 1 \\
0 & 1 & 1 & 0 & 0 & 0
\end{array}\right) .
$$

If the incidence matrix is replaced with

$$
B=\left(\begin{array}{cccccc}
0 & 0 & 0 & 0 & 0.9 & 0 \\
0 & 0 & 0 & 0 & 0.9 & 0.7 \\
0.7 & 0 & 0 & 0 & 0 & 0.9 \\
0.6 & 0 & 0 & 0 & 0 & 0 \\
0.9 & 0.85 & 0 & 0 & 0 & 0 \\
0 & 0 & 0.95 & 0 & 0 & 0 \\
0 & 0 & 0.95 & 0.9 & 0 & 1 \\
0 & 0.95 & 0.95 & 0 & 0 & 0
\end{array}\right),
$$

then the uncertain incidence matrix B implies a new type of uncertain hypergraph with uncertain vertex-hyperedge relationships. 
Generally, another type of uncertainty may actually exist in hypergraphs in the case that both the adjacency relationships among the vertices and the incidence relationships between the vertices and the hyperedges are uncertain.

Definition 11. A double uncertain hypergraph is defined as a quaternary system $H=\left(V, E, \psi, \psi^{\prime}\right)$ where $V$ is the vertex set, $E$ is the hyperedge set of $H, \psi: E \rightarrow[0,1]$ is a set function in which $\psi(e)$ assigns the uncertain measure of the existence of the hyperedge $e \in E$ and $\psi^{\prime}: V \times E \rightarrow[0,1]$ is a set function in which $\psi^{\prime}((v, e))$ assigns the uncertain measure of the incidence existence between vertex $v \in V$ and hyperedge $e \in E$.

In this article, we restrictively focus on the first type of uncertain hypergraphs which have uncertain topology adjacency structures.

\section{Operations of Uncertain Hypergraphs}

Definition 12. Given two uncertain hypergraphs $H_{1}=\left(V_{1}, E_{1}, \psi_{1}\right)$ and $H_{2}=\left(V_{2}, E_{2}, \psi_{2}\right)$, $H_{1}$ is said to be a subgraph of $H_{2}$ if $V_{1} \subseteq V_{2}, E_{1} \subseteq E_{2}$ and $\psi_{1}=\left.\psi_{2}\right|_{E_{1}}$. We write $H_{1} \subseteq H_{2}$. Alternatively, we also call $H_{2}$ a supergraph of $H_{1}$, denoted by $H_{2} \supseteq H_{1}$.

The isomorphism relationship of uncertain hypergraphs is an important relationship between uncertain hypergraphs.

Definition 13. An uncertain hypergraph $H_{1}=\left(V_{1}, E_{1}, \psi_{1}\right)$ is isomorphic to the other uncertain hypergraph $\mathrm{H}_{2}=\left(V_{2}, E_{2}, \psi_{2}\right)$, written $H_{1} \cong H_{2}$, if there exists a bijection $f: V_{1} \rightarrow$ $V_{2}$ and a bijection $g: E_{1} \rightarrow E_{2}$ such that: 1) $\forall e=\left\{v_{1}, v_{2}, \cdots, v_{k}\right\}, e \in E_{1}$ iff $g(e)=$ $\left\{f\left(v_{1}\right), f\left(v_{2}\right), \cdots, f\left(v_{k}\right)\right\} \in E_{2}$ and 2) $\psi_{1}(e)=\psi_{2}(g(e))$.

Theorem 1. Two simple uncertain hypergraphs $H_{1}=\left(V_{1}, E_{1}, \psi_{1}\right)$ and $H_{2}=\left(V_{2}, E_{2}, \psi_{2}\right)$ are said to be isomorphic iff there exists a bijection $f: V_{1} \rightarrow V_{2}$ such that: 1) $\forall e=\left\{v_{1}, v_{2}, \cdots, v_{k}\right\}$, $e \in E_{1}$ iff $\left\{f\left(v_{1}\right), f\left(v_{2}\right), \cdots, f\left(v_{k}\right)\right\} \in E_{2}$ and 2) $\psi_{1}(e)=\psi_{2}\left(\left\{f\left(v_{1}\right), f\left(v_{2}\right), \cdots, f\left(v_{k}\right)\right\}\right)$.

Proof. Assume $H_{1}$ and $H_{2}$ are two isomorphic uncertain hypergraphs, i.e., $H_{1} \cong H_{2}$. It follows from Definition 13 that (1) and (2) can be easily verified. Conversely, suppose

that $f: V_{1} \rightarrow V_{2}$ is a bijection satisfying (1) and (2). Now, we construct a mapping $g: E_{1} \rightarrow E_{2}$ satisfying that for each hyperedge $e=\left\{v_{1}, v_{2}, \cdots, v_{k}\right\} \in E_{1}$, we have $g(e)=$ $\left\{f\left(v_{1}\right), f\left(v_{2}\right), \cdots, f\left(v_{k}\right)\right\} \in E_{2}$. Since $H_{1}$ and $H_{2}$ are simple graphs, it follows from (1) that $g$ is a bijection. That is, each $e=\left\{v_{1}, v_{2}, \cdots, v_{k}\right\} \in E_{1}$ iff $g(e)=\left\{f\left(v_{1}\right), f\left(v_{2}\right), \cdots, f\left(v_{k}\right)\right\} \in$ $E_{2}$. Combining with (2), the proof is completed.

We now introduce the complement, union and joint operations on uncertain hypergraphs as follows:

Definition 14. The complement of the uncertain hypergraph $H=(V, E, \psi)$ is an uncertain hypergraph $H^{c}=\left(V^{c}, E^{c}, \psi^{c}\right)$ where $V^{c}=V, E^{c}=E$ and $\psi^{c}: E^{c} \rightarrow[0,1]$ satisfying that $\psi^{c}(e)=1-\psi(e)$ for each $e \in E$.

Theorem 2. Let $H=(V, E, \psi)$ be a simple uncertain hypergraph. Then for each hyperedge e $\in E$, we have

$$
\psi_{\left(H^{c}\right)^{c}}(e)=\psi_{H}(e) .
$$


Proof. Obviously, $V\left(\left(H^{c}\right)^{c}\right)=V\left(H^{c}\right)=V(H)$. In addition, for each hyperedge $e \in E$,

$$
\begin{aligned}
\psi_{\left(H^{c}\right)^{c}}(e) & =1-\psi_{\left(H^{c}\right)}(e) \\
& =1-\left(1-\psi_{H}(e)\right) \\
& =\psi_{H}(e) .
\end{aligned}
$$

The proof is completed.

Definition 15. Given two uncertain hypergraphs $H_{1}=\left(V_{1}, E_{1}, \psi_{1}\right)$ and $H_{2}=\left(V_{2}, E_{2}, \psi_{2}\right)$, the union $\mathrm{H}_{1} \cup \mathrm{H}_{2}$ of the uncertain hypergraphs $\mathrm{H}_{1}$ and $\mathrm{H}_{2}$ is an uncertain hypergraph $H=(V, E, \psi)$ where $V=V_{1} \cup V_{2}, E=E_{1} \cup E_{2}$ and $\psi: E \rightarrow[0,1]$, satisfying that

$$
\psi(e)= \begin{cases}\psi_{1}(e), & \text { if } e \in E_{1} \\ \psi_{2}(e), & \text { if } e \in E_{2} .\end{cases}
$$

Definition 16. The joint $H_{1} \cap H_{2}$ of the uncertain hypergraphs $H_{1}$ and $H_{2}$ is an uncertain hypergraph $H=(V, E, \psi)$ where $V=V_{1} \cap V_{2}, E=E_{1} \cap E_{2}$ and $\psi: E \rightarrow[0,1]$ satisfying that $\psi(e)=\psi_{1}(e)=\psi_{2}(e)$ for each hyperedge $e \in E$.

Theorem 3. Let $H_{1}=\left(V_{1}, E_{1}, \psi_{1}\right)$ and $H_{2}=\left(V_{2}, E_{2}, \psi_{2}\right)$ be two simple uncertain hypergraphs. Then the following properties hold: (1) $\left(H_{1} \cup H_{2}\right)^{c} \cong H_{1}^{c} \cup H_{2}^{c}$ and (2) $\left(H_{1} \cap H_{2}\right)^{c} \cong H_{1}^{c} \cap H_{2}^{c}$.

Proof. (1) Let $I: V_{1} \cup V_{2} \rightarrow V_{1} \cup V_{2}$ be an identity map on vertex set $V_{1} \cup V_{2}$. Obviously, $e=\left\{v_{1}, v_{2}, \cdots, v_{k}\right\}$ is a hyperedge of $\left(H_{1} \cup H_{2}\right)^{c}$ iff $\left\{I\left(v_{1}\right), I\left(v_{2}\right), \cdots, I\left(v_{k}\right)\right\}$ is a hyperedge of $H_{1}^{c} \cup H_{2}^{c}$.

Note that

$$
\begin{aligned}
\psi_{\left(H_{1} \cup H_{2}\right)^{c}}(e) & =1-\psi_{H_{1} \cup H_{2}}(e) \\
& =\left\{\begin{array}{l}
1-\psi_{1}(e), \quad \text { if } e \in E_{1} \\
1-\psi_{2}(e), \quad \text { if } e \in E_{2}
\end{array}\right. \\
& =\psi_{H_{1}^{c} \cup H_{2}^{c}}(e)=\psi_{H_{1}^{c} \cup H_{2}^{c}}\left(I\left(v_{1}\right), I\left(v_{2}\right), \cdots, I\left(v_{k}\right)\right) .
\end{aligned}
$$

It follows from Theorem 1 that $\left(H_{1} \cup H_{2}\right)^{c} \cong H_{1}^{c} \cup H_{2}^{c}$.

(2) Let $I: V_{1} \cap V_{2} \rightarrow V_{1} \cap V_{2}$ be an identity map on vertex set $V_{1} \cap V_{2}$. Obviously, $e=\left\{v_{1}, v_{2}, \cdots, v_{k}\right\}$ is a hyperedge of $\left(H_{1} \cap H_{2}\right)^{c}$ iff $\left\{I\left(v_{1}\right), I\left(v_{2}\right), \cdots, I\left(v_{k}\right)\right\}$ is a hyperedge of $H_{1}^{c} \cap H_{2}^{c}$.

Note that

$$
\begin{aligned}
\psi_{\left(H_{1} \cap H_{2}\right)^{c}}(e) & =1-\psi_{H_{1} \cap H_{2}}(e) \\
& =1-\psi_{1}(e)=1-\psi_{2}(e) \\
& =\psi_{H_{1}^{c} \cap H_{2}^{c}}(e)=\psi_{H_{1}^{c} \cap H_{2}^{c}}\left(I\left(v_{1}\right), I\left(v_{2}\right), \cdots, I\left(v_{k}\right)\right) .
\end{aligned}
$$

It follows from Theorem 1 that $\left(H_{1} \cap H_{2}\right)^{c} \cong H_{1}^{c} \cap H_{2}^{c}$.

In the following, we give a definition of the $\alpha$-cut to describe the relationship between a hypergraph and an uncertain hypergraph:

Definition 17. The $\alpha$-cut $H_{\alpha}$ of an uncertain hypergraph $H=(V, E, \psi)$ is defined as a certain hypergraph $H_{\alpha}=\left(V_{\alpha}, E_{\alpha}\right)$ where $V_{\alpha}=V$ is the vertex set, $E_{\alpha}$ is the hyperedge set of $H_{\alpha}$ such that $e \in E_{\alpha}$ iff $e \in E$ and $\psi(e) \geq \alpha$. 
It is not so difficult to prove that the $\alpha$-cuts of the union and joint operations of two uncertain hypergraphs $H_{1}$ and $H_{2}$ have the following properties:

Theorem 4. Given two uncertain hypergraphs $H_{1}=\left(V_{1}, E_{1}, \psi_{1}\right)$ and $H_{2}=\left(V_{2}, E_{2}, \psi_{2}\right)$, we have (1) $\left(H_{1} \cup H_{2}\right)_{\alpha}=\left(H_{1}\right)_{\alpha} \cup\left(H_{2}\right)_{\alpha}$ and (2) $\left(H_{1} \cap H_{2}\right)_{\alpha}=\left(H_{1}\right)_{\alpha} \cap\left(H_{2}\right)_{\alpha}$.

Proof. For a given $\alpha \in(0,1)$, we will corroborate the results as follows:

(1) Obviously, $\left(H_{1} \cup H_{2}\right)_{\alpha}$ and $\left(H_{1}\right)_{\alpha} \cup\left(H_{2}\right)_{\alpha}$ have the same vertex set $V_{1} \cup V_{2}$.

On the other hand, we will prove that $e$ is a hyperedge of $\left(H_{1} \cup H_{2}\right)_{\alpha}$ iff $e$ is a hyperedge of $\left(H_{1}\right)_{\alpha} \cup\left(H_{2}\right)_{\alpha}$.

If $e$ is a hyperedge of $\left(H_{1} \cup H_{2}\right)_{\alpha}$, then $e \in E_{1} \cup E_{2}$ such that $\psi_{1}(e) \geq \alpha$ or $\psi_{2}(e) \geq \alpha$. More precisely, we have $e \in E_{1}, \psi_{1}(e) \geq \alpha$, or $e \in E_{2}, \psi_{2}(e) \geq \alpha$. Thus, in either case, $e$ is always a hyperedge of $\left(H_{1}\right)_{\alpha} \cup\left(H_{2}\right)_{\alpha}$. Vice versa, it is easy to verify that if $e$ is a hyperedge of $\left(H_{1}\right)_{\alpha} \cup\left(H_{2}\right)_{\alpha}$, then it is also a hyperedge of $\left(H_{1} \cup H_{2}\right)_{\alpha}$. Thus, we have $\left(H_{1} \cup H_{2}\right)_{\alpha}=$ $\left(H_{1}\right)_{\alpha} \cup\left(H_{2}\right)_{\alpha}$.

(2) Obviously, $\left(H_{1} \cap H_{2}\right)_{\alpha}$ and $\left(H_{1}\right)_{\alpha} \cap\left(H_{2}\right)_{\alpha}$ have the same vertex set $V_{1} \cap V_{2}$. On the other hand, we will prove that $e$ is a hyperedge of $\left(H_{1} \cap H_{2}\right)_{\alpha}$ iff $e$ is a hyperedge of $\left(H_{1}\right)_{\alpha} \cap\left(H_{2}\right)_{\alpha}$.

If $e$ is a hyperedge of $\left(H_{1} \cap H_{2}\right)_{\alpha}$, then $e \in E_{1} \cap E_{2}, \psi_{1}(e) \geq \alpha$ and $\psi_{2}(e) \geq \alpha$. More precisely, we have $e \in E_{1}, \psi_{1}(e) \geq \alpha$ and $e \in E_{2}, \psi_{2}(e) \geq \alpha$. In other words, $e \in\left(H_{1}\right)_{\alpha}$ and $e \in\left(H_{2}\right)_{\alpha}$ both hold. In accordance with Definition 16, $e$ is a hyperedge of $\left(H_{1}\right)_{\alpha} \cap\left(H_{2}\right)_{\alpha}$. A similar process may prove that if $e$ is a hyperedge of $\left(H_{1}\right)_{\alpha} \cap\left(H_{2}\right)_{\alpha}$, then it is also a hyperedge of $\left(H_{1} \cap H_{2}\right)_{\alpha}$. Thus, we have $\left(H_{1} \cap H_{2}\right)_{\alpha}=\left(H_{1}\right)_{\alpha} \cap\left(H_{2}\right)_{\alpha}$.

\section{Some Topological Indexes of Uncertain Hypergraphs}

By topological indices, we mean the numerical parameters of an uncertain hypergraph which characterize its topology structure. We are very interested in some questions of uncertain hypergraphs similar to the concepts and solutions in graphs or hypergraphs.

\subsection{Degrees in Uncertain Hypergraphs}

Definition 18. Let $H=(V, E, \psi)$ be an uncertain hypergraph with vertices $V=\left\{v_{1}, v_{2}, \cdots, v_{n}\right\}$ and hyperedges $E=\left\{e_{1}, e_{2}, \cdots, e_{m}\right\}$. For a vertex $v_{i} \in V$, the star $H\left(v_{i}\right)$ centered in $v_{i}$ is the set of hyperedges containing $v_{i}$. That is to say, $H\left(v_{i}\right)=\left\{e \mid v_{i} \in e\right\}=\left\{e_{1}, e_{2}, \cdots, e_{n_{i}}\right\}$. Then $d_{H}\left(v_{i}\right)=\sum_{j=1}^{n_{i}} \psi\left(e_{j}\right)$ is called the degree of $v_{i}$. The number $d(H)=\frac{1}{n} \sum_{i=1}^{n} d_{H}\left(v_{i}\right)$ is called the average degree of the uncertain hypergraph $H$.

If each vertex has the same degree, we say that the uncertain hypergraph is regular, or we say it is $k$-regular if for every $v \in V, d_{H}(v)=k$. The maximum degree $\max _{v \in V} d_{H}(v)$ of an uncertain hypergraph $H$ is denoted by $\Delta(H)$. The minimum degree $\min _{v \in V} d_{H}(v)$ of an uncertain hypergraph $H$ is denoted by $\delta(H)$. Clearly, we have $\delta(H) \leq d(H) \leq \Delta(H)$.

Example 8. Let $H=(V, E)$ be a hypergraph with vertices $V=\left\{v_{1}, v_{2}, v_{3}, v_{4}, v_{5}, v_{6}, v_{7}\right\}$ and hyperedges $E=\left\{e_{1}, e_{2}, e_{3}, e_{4}\right\}=\left\{\left\{v_{1}, v_{2}, v_{3}\right\},\left\{v_{2}, v_{3}\right\},\left\{v_{3}, v_{5}, v_{6}\right\},\left\{v_{4}, v_{6}\right\}\right\}$. Define $\psi\left(e_{1}\right)=0.85, \psi\left(e_{2}\right)=0.90, \psi\left(e_{3}\right)=0.95$ and $\psi\left(e_{4}\right)=1$. Then $H=(V, E, \psi)$ is an uncertain hypergraph in which the degrees $d_{H}\left(v_{1}\right)=0.85, d_{H}\left(v_{2}\right)=1.75, d_{H}\left(v_{3}\right)=2.70, d_{H}\left(v_{4}\right)=$ $1, d_{H}\left(v_{5}\right)=0.95, d_{H}\left(v_{6}\right)=1.95$ and $d_{H}\left(v_{7}\right)=0$, respectively. Thus $\Delta(H)=2.70$ and $\delta(H)=0$. The average degree of the uncertain hypergraph $H$ is $d(H)=1.31$.

Example 9. Let $H=(V, E)$ be a graph with vertex set $V=\left\{v_{1}, v_{2}, v_{3}, v_{4}, v_{5}, v_{6}\right\}$ and edge set $E=\left\{\left\{v_{1}, v_{2}\right\},\left\{v_{2}, v_{3}\right\},\left\{v_{3}, v_{4}\right\},\left\{v_{4}, v_{5}\right\},\left\{v_{5}, v_{6}\right\},\left\{v_{6}, v_{1}\right\}\right\}$. Define $\psi\left(e_{1}\right)=0.91, \psi\left(e_{2}\right)=$ $0.92, \psi\left(e_{3}\right)=0.91, \psi\left(e_{4}\right)=0.92, \psi\left(e_{5}\right)=0.91$ and $\psi\left(e_{6}\right)=0.92$. Then $H=(V, E, \psi)$ is a 1.83-regular uncertain hypergraph. 


\subsection{Distances in Uncertain Hypergraphs}

It is natural to consider the distance between two vertices in an uncertain hypergraph. Since the hyperedges exist with uncertain measure, the concept of path in an uncertain hypergraph is also related to uncertain measure.

Definition 19. Let $H=(V, E, \psi)$ be an uncertain hypergraph without an isolated vertex. An uncertain path $P$ in $H$ from $x$ to $y$ is a vertex-hyperedge alternative sequence $x=v_{1} e_{1} v_{2} e_{2} \cdots v_{s} e_{s} v_{s+1}=$ $y$ such that

(1) $v_{1}, v_{2}, \cdots, v_{s}, v_{s+1}$ are distinct vertices with the possibility that $v_{1}=v_{s+1}$ if $x=y$;

(2) $e_{1}, e_{2}, \cdots, e_{s}$ are distinct hyperedges;

(3) Each hyperedge $e_{i}$ has corresponding uncertain measure $\psi\left(e_{i}\right)$ for $i=1,2, \cdots, s$;

(4) $v_{i}, v_{i+1} \in e_{i}$ for $i=1,2, \cdots$, s.

The integer $s$ is the length of the uncertain path $P$. Denote $\alpha=\min _{e_{i} \in P}\left\{\psi\left(e_{i}\right)\right\}$ and $\beta=1-\max _{e_{i} \in P}\left\{\psi\left(e_{i}\right)\right\}$. Then the uncertain path $P$ is called the $\alpha$-maximum belief degree path from $x$ to $y$, or antisymmetrically, it is also called the $\beta$-minimum risk path from $x$ to $y$. The uncertain path $P$ is called an uncertain cycle if $x=v_{1}=v_{s+1}=y$.

Notice that if there is an uncertain path with $\alpha$-maximum belief degree from $x$ to $y$, then there is also an uncertain path with $\alpha$-maximum belief degree from $y$ to $x$. In this case, we say that the uncertain path $P$ connects $x$ and $y$ with $\alpha$-maximum belief degree. An uncertain hypergraph is $\alpha$-connected if for any pair of vertices, there is an uncertain path with $\alpha$-maximum belief degree which connects these vertices; otherwise, it is not $\alpha$ connected, which is also called $\alpha$-disconnected. It is obvious that if an uncertain hypergraph $H$ is $\alpha$-connected and $\alpha \geq \gamma$, then $H$ is $\gamma$-connected.

We define the relation $x R y$ with $\alpha$-maximum belief degree if and only if either there is an uncertain path with $\alpha$-maximum belief degree from $x$ to $y$ or $x=y$. Then relation $R$ is an equivalence relation, which can classify the equivalence classes of this relation into the $\alpha$-connected components of the uncertain hypergraph.

In a hypergraph, the distance between two vertices is the minimal number of hyperedges that connect the two vertices. By contrast, the $\alpha$-belief degree distance between two vertices in an uncertain hypergraph is the minimal number of hyperedges that connect the two vertices at $\alpha$-belief degree level.

What we concern ourselves about here is the topological existence of a belief degree path between $x$ and $y$ other than the physical distance between the two vertices. There may exist a not unique path with $\alpha$-maximum belief degree from $x$ to $y$ in an uncertain hypergraph.

Definition 20. Let $H=(V, E, \psi)$ be an uncertain hypergraph. The distance $d_{\alpha}(x, y)$ at $\alpha$-belief degree level between two vertices $x$ and $y$ is the minimum length of a path with $\alpha$-maximum belief degree which connects $x$ and $y$. The diameter diam $m_{\alpha}(H)$ at $\alpha$-belief degree level of $H$ is defined by $\operatorname{diam}_{\alpha}(H)=\max \left\{d_{\alpha}(x, y) \mid x, y \in V\right\}$.

If there is a pair of vertices $x, y$ with no path from $x$ to $y$ at $\alpha$-belief degree level (or from $y$ to $x$ ), we define $d_{\alpha}(x, y)=\infty$. An $\alpha$-connected component is a maximal set of vertices $X \subseteq V$ such that $d_{\alpha}(x, y) \neq \infty$ for all $x, y \in X$.

Example 10. Let $H=(V, E, \psi)$ be an uncertain hypergraph with vertex set $V=\left\{v_{1}, v_{2}, v_{3}, v_{4}\right.$, $\left.v_{5}, v_{6}, v_{7}, v_{8}, v_{9}, v_{10}\right\}$ and edge set

$$
E=\left\{\left\{v_{1}, v_{2}\right\},\left\{v_{2}, v_{3}, v_{4}\right\},\left\{v_{4}, v_{5}\right\},\left\{v_{3}, v_{8}\right\},\left\{v_{5}, v_{10}\right\},\left\{v_{6}, v_{7}, v_{8}, v_{9}, v_{10}\right\}\right\} .
$$

Define $\psi\left(e_{1}\right)=0.91, \psi\left(e_{2}\right)=0.92, \psi\left(e_{3}\right)=0.93, \psi\left(e_{4}\right)=0.94, \psi\left(e_{5}\right)=0.95$ and $\psi\left(e_{6}\right)=0.96$. It is given that $\alpha=0.9$. Then, we have $d_{\alpha}\left(v_{1}, v_{2}\right)=1, d_{\alpha}\left(v_{1}, v_{3}\right)=2, d_{\alpha}\left(v_{1}, v_{4}\right)=2, d_{\alpha}\left(v_{1}, v_{5}\right)=$ $3, d_{\alpha}\left(v_{1}, v_{6}\right)=4, d_{\alpha}\left(v_{1}, v_{7}\right)=4, d_{\alpha}\left(v_{1}, v_{8}\right)=3, d_{\alpha}\left(v_{1}, v_{9}\right)=4$ and $d_{\alpha}\left(v_{1}, v_{10}\right)=4$. 


\subsection{Reliability Indexes of Uncertain Hypergraphs}

The reliability index of the topological structure of an uncertain hypergraph is a topic of our interest and concern. It is also the starting point of reliability analysis for uncertain hypergraphs based on uncertainty theory. Note that uncertain reliability analysis and uncertain risk analysis have duality in mathematics.

Definition 21. Assume $H=(V, E, \psi)$ is an uncertain hypergraph of order $n$ with $V=\left\{v_{1}, v_{2}\right.$, $\left.\cdots, v_{n}\right\}, E=\left(e_{1}, e_{2}, \cdots, e_{m}\right)$ and $\psi(E)=\left(\psi\left(e_{1}\right), \psi\left(e_{2}\right), \cdots, \psi\left(e_{m}\right)\right)$. Then the minimum adjacency belief degree

$$
R\left(\psi\left(e_{1}\right), \psi\left(e_{2}\right), \cdots, \psi\left(e_{m}\right)\right)=\bigwedge_{i=1}^{m} \psi\left(e_{i}\right)
$$

is called the infimum adjacency reliability index, and the maximum adjacency belief degree

$$
R\left(\psi\left(e_{1}\right), \psi\left(e_{2}\right), \cdots, \psi\left(e_{m}\right)\right)=\bigvee_{i=1}^{m} \psi\left(e_{i}\right)
$$

is called the supremum adjacency reliability index.

Dually, the maximum adjacency belief degree

$$
R^{\prime}\left(\psi\left(e_{1}\right), \psi\left(e_{2}\right), \cdots, \psi\left(e_{m}\right)\right)=1-\bigwedge_{i=1}^{m} \psi\left(e_{i}\right)
$$

is called the supremum adjacency risk index, and the minimum adjacency belief degree

$$
R^{\prime}\left(\psi\left(e_{1}\right), \psi\left(e_{2}\right), \cdots, \psi\left(e_{m}\right)\right)=1-\bigvee_{i=1}^{m} \psi\left(e_{i}\right)
$$

is called the infimum adjacency risk index.

For instance, let $H=(V, E, \psi)$ be an uncertain hypergraph as shown in Example 10. Then the infimum adjacency reliability index

$$
R\left(\psi\left(e_{1}\right), \psi\left(e_{2}\right), \cdots, \psi\left(e_{6}\right)\right)=\bigwedge_{i=1}^{6} \psi\left(e_{i}\right)=0.91 ;
$$

the supremum adjacency reliability index

$$
R\left(\psi\left(e_{1}\right), \psi\left(e_{2}\right), \cdots, \psi\left(e_{6}\right)\right)=\bigvee_{i=1}^{6} \psi\left(e_{i}\right)=0.96 ;
$$

the supremum adjacency risk index

$$
R^{\prime}\left(\psi\left(e_{1}\right), \psi\left(e_{2}\right), \cdots, \psi\left(e_{6}\right)\right)=1-\bigwedge_{i=1}^{6} \psi\left(e_{i}\right)=0.09
$$

and the infimum adjacency risk index

$$
R^{\prime}\left(\psi\left(e_{1}\right), \psi\left(e_{2}\right), \cdots, \psi\left(e_{6}\right)\right)=1-\bigvee_{i=1}^{6} \psi\left(e_{i}\right)=0.04
$$




\subsection{Entropy of Uncertain Hypergraphs}

Entropy is an important concept which provides a quantitative measurement for the degree of uncertainty of an uncertain hypergraph. We introduce below the notion of entropy associated with an uncertain hypergraph.

Definition 22. Let $H=(V, E, \psi)$ with $V=\left\{v_{1}, v_{2}, \cdots, v_{n}\right\}$ be an uncertain hypergraph. Then we can define the entropy $I(H)$ of $H$ by

$$
I(H)=-\sum_{i=1}^{n} \lambda_{i} \ln \lambda_{i}
$$

where $\lambda_{i}=\frac{d_{i}}{\sum_{j=1}^{n} d_{j}}$ and $d_{i}=\sum_{v_{i} \in e} \psi(e)$ is the degree of the vertex $v_{i}$ for $i=1,2, \cdots, n$.

Example 11. Let $H=(V, E, \psi)$ be an uncertain hypergraph with vertex set $V=\left\{v_{1}, v_{2}, v_{3}, v_{4}\right.$, $\left.v_{5}, v_{6}\right\}$ and edge set

$$
E=\left\{\left\{v_{1}, v_{2}\right\},\left\{v_{2}, v_{3}, v_{4}\right\},\left\{v_{3}, v_{4}, v_{5}, v_{6}\right\}\right\} .
$$

Define $\psi\left(e_{1}\right)=0.91, \psi\left(e_{2}\right)=0.92$ and $\psi\left(e_{3}\right)=0.93$. It is easy to verify that $d_{1}=0.91$, $d_{2}=1.83, d_{3}=d_{4}=1.85$ and $d_{5}=d_{6}=0.93$. Then we have

$$
I(H)=-\sum_{i=1}^{6} \lambda_{i} \ln \lambda_{i}=1.74
$$

\section{Conclusions and Future Work}

Graphs, uncertain graphs, hypergraphs and uncertain hypergraphs are important and useful concepts or tools in describing systems in our real lives. In our view, on one side, a graph is used to describe a system with relative simplicity, while a hypergraph is used to describe a system with relative complexity. On the other side, a graph is used to describe a system with determinacy, while an uncertain graph is used to describe a system with uncertainty. In a more profound view, an uncertain graph is used to describe a system with indeterminacy and relative simplicity, while an uncertain hypergraph is used to describe a system with uncertainty and relative complexity.

The main contributions of this paper can be summarized as follows: Firstly, we constructed the foundation for a new interdisciplinary theory for uncertainty theory and hypergraph theory termed uncertain hypergraph theory. The concept of an uncertain hypergraph was proposed and the representations of an uncertain hypergraph were discussed. Subsequently, the operations of uncertain hypergraphs were studied, and some properties were verified. Finally, some topological indexes were introduced. The importance and originality of this study are that we hope this work can serve as an opportunity of reflection for experienced complexity scientists and experienced uncertainty scientists as well as an introductory resource for new researchers.

Some directions for future work are described here: As part of our future work, we would first like to introduce the uncertain hypernetwork, which is different from the hypergraph in some aspects. Second, we would like to study ways in which we could improve the computational efficiency of the proposed methods. Third, we would like to think about hypergraph theory under more a complex environment, such as an uncertain random environment. Chance theory was introduced by Liu [33] to model complex systems related to uncertainty and randomness. Hopefully, the related topics, such as connectivity, regularity and operations on hypergraphs, can be further studied in the future within the framework of chance theory.

Author Contributions: Conceptualization, J.P.; methodology, J.P. and B.Z.; investigation, J.P. and B.Z.; writing — original draft preparation, J.P.; writing — review and editing, J.P. and K.A.S. All authors have read and agreed to the published version of the manuscript. 
Funding: This work was supported by the National Natural Science Foundation of China (Grant Nos. 61873108, 61703438 and 72071092 ).

Conflicts of Interest: The authors declare no conflict of interest.

\section{References}

1. Bondy, J.A.; Murty, U.S.R. Graph Theory with Application; Elsevier: NewYork, NY, USA, 1976.

2. Berge, C. Graphs and Hypergraphs; North-Holland: Amsterdam, The Netherlands, 1973.

3. Dankelmann, P.; Meierling, D. Maximally edge-connected hypergraphs. Discrete Math. 2016, 1, 33-38. [CrossRef]

4. Zhao, S.; Meng, J. Sufficient conditions for hypergraphs to be maximally edge-connected. Appl. Math. Comput. 2018, 333, 362-368. [CrossRef]

5. Frankl, P. Maximum degree and diversity in intersecting hypergraphs. J. Combin. Theory Ser. B 2020, 144, 81-94. [CrossRef]

6. Hàn, H.; Han, J.; Zhao, Y. Minimum degree thresholds for Hamilton (k/2)-cycles in $k$-uniform hypergraphs. J. Combin. Theory Ser. B 2022, 153, 105-148.

7. Yu, G.; Yan, C.; Sun, L.; Wu, Y.; Zhang, H. Spectral radius and matching number of the unicyclic hypergraph. Linear Algebra Appl. 2021, 610, 571-590. [CrossRef]

8. Lu, H.; Yu, X.; Yuan, X. Rainbow matchings for 3-uniform hypergraphs. J. Combin. Theory Ser. A 2021, 183, 105489. [CrossRef]

9. Kolmogorov, A. Grundbegriffe der Wahrscheinlichkeitsrechnung; Springer: Berlin, Germany, 1933.

10. Erdös, P.; Rényi, A. On random graph I. Publ. Math. 1959, 6, 290-297.

11. Erdös, P.; Rényi, A. On the strength of connectedness of a random graph. Acta Math. Hungar. 1964, 12, $261-267$.

12. Gilbert, E. Random graphs. Ann. Math. Stat. 1959, 30, 1141-1144. [CrossRef]

13. Cooper, J. Adjacency spectra of random and complete hypergraphs. Linear Algebra Appl. 2020, 596, 184-202. [CrossRef]

14. Semenov, A.; Shabanov, D. On the weak chromatic number of random hypergraphs. Discrete Appl. Math. 2020, 276 , 134-154. [CrossRef]

15. Liu, Y.; Zhao, Y. On the upper tail problem for random hypergraphs. Random Struct. Algor. 2021, 58, 179-220. [CrossRef]

16. Zadeh, L.A. Fuzzy sets. Inform. Control 1965, 8, 338-353. [CrossRef]

17. Rosenfeld, A. Fuzzy graphs. In Fuzzy Sets and Their Applications to Cognitive and Decision Processes; Academic: New York, NY, USA, 1975; pp. 77-95.

18. Wang, Q.; Gong, Z. An application of fuzzy hypergraphs and hypergraphs in granular computing. Inform. Sci. 2018, 429, 296-314. [CrossRef]

19. Luqman, A.; Akram, M.; Al-Kenani, A.; Alcantud, J. A study on hypergaph representations of complex fuzzy information. Symmetry 2019, 11, 1381. [CrossRef]

20. Sarwar, M.; Akram, M.; Shahzadi, S. Bipolar fuzzy soft information applied to hypergraphs. Soft Comput. 2021, 25, 3417-3439. [CrossRef]

21. Akram, M.; Luqman, A. Fuzzy Hypergraphs and Related Extensions; Springer: Singapore, 2020.

22. Liu, B. Uncertainty Theory, 2nd ed.; Springer: Berlin, Germany, 2007.

23. Liu, B. Uncertainty Theory: A Branch of Mathematics for Modeling Human Uncertainty; Springer: Berlin, Germany, 2010.

24. Gao, X.; Gao, Y. Connectedness index of uncertain graphs. Internat. J. Uncertain. Fuzziness Knowl. Based Syst. 2013, 21, 127-137. [CrossRef]

25. Zhang, B.; Peng, J. Euler index in uncertain graph. Appl. Math. Comput. 2012, 218, 10279-10288. [CrossRef]

26. Zhang, B.; Peng, J. Matching index of an uncertain graph: Concept and algorithm. Appl. Comput. Math. $2013,12,381-391$.

27. Zhang, B.; Peng, J. Connectedness strength of two vertices in an uncertain graph. Int. J. Comput. Math. 2013, 90, 246-257. [CrossRef]

28. Gao, X. Regularity index of uncertain graph. J. Intell. Fuzzy Syst. 2014, 4, 1671-1678. [CrossRef]

29. Gao, Y.; Qin, Z. On computing the edge-connectivity of an uncertain graph. IEEE Trans. Fuzzy Syst. 2016, 24, 981-991. [CrossRef]

30. Bretto, A. Hypergraph Theory; Springer: Heidelberg, Germany, 2013.

31. Liu, B. Some research problems in uncertainty theory. J. Uncertain Syst. 2009, 3, 3-10.

32. Liu, B. Uncertainty Theory, 4th ed.; Springer: Berlin, Germany, 2015.

33. Liu, Y. Uncertain random variables: A mixture of uncertainty and randomness. Soft Comput. 2013, 17, 625-634. [CrossRef] 